\title{
A Measurement Study of White Spaces Across Diverse Population Densities
}

\author{
Pengfei Cui, Hui Liu, Dinesh Rajan, and Joseph Camp \\ Department of Electrical Engineering, Southern Methodist University, Dallas, TX
}

\begin{abstract}
While many metropolitan areas sought to deploy city-wide WiFi networks, the densest urban areas were not able to broadly leverage the technology for large-scale Internet access. Ultimately, the small spatial separation required for effective 802.11 links in these areas resulted in prohibitively large upfront costs. The FCC has reapportioned spectrum from TV white spaces for the purposes of large-scale Internet connectivity via wireless topologies of all kinds. The far greater range of these lower carrier frequencies are especially critical in rural areas, where high levels of aggregation could dramatically lower the cost of deployment and is in direct contrast to dense urban areas in which networks are built to maximize spatial reuse. Thus, leveraging a broad range of spectrum across diverse population densities becomes a critical issue for the deployment of data networks with WiFi and white space bands. In this paper, we measure the spectrum utility in the Dallas-Fort Worth metropolitan and surrounding areas and propose a measurement-driven band selection framework, Multiband Access Point Estimation (MAPE). In particular, we study the white space and WiFi bands with in-field spectrum utility measurements, revealing the number of access points required for an area with channels in multiple bands. In doing so, we find that networks with white space bands reduce the number of access points by up to $1650 \%$ in sparse rural areas over similar WiFi-only solutions. In more populated rural areas and sparse urban areas, we find an access point reduction of $660 \%$ and $412 \%$, respectively. However, due to the heavy use of white space bands in dense urban areas, the cost reductions invert (an increase in required access points of $6 \%$ ). Finally, we numerically analyze band combinations in typical rural and urban areas and show the critical factor that leads to cost reduction: considering the same total number of channels, as more channels are available in the white space bands, less access points are required for a given area.
\end{abstract}

\section{INTRODUCTION}

The FCC has approved the use of broadband services in the white spaces of UHF TV bands, which were formerly exclusively licensed to television broadcasters. These white space bands are now available for unlicensed public use, enabling the deployment of wireless access networks across a broad range of scenarios from sparse rural areas (one of the key applications identified by the FCC) to dense urban areas [1]. The white space bands operate in available channels from 54-806 MHz, having a far greater propagation range than WiFi bands for similar transmission power [2].

Specific to rural areas, the lack of user density and corresponding traffic demand per unit area as compared to dense urban areas allows greater levels of spatial aggregation to reduce the total number of required access points, lowering network deployment costs. In densely populated urban areas, the greater concentration of users and higher levels of traffic demand can be served by maximizing the spatial reuse. While many works have worked to address multihop wireless network deployment in terms of maximizing served user demand and/or minimizing network costs, the unique propagation characteristics and the interference from coexisting activities in white space bands have either not been jointly studied or assumed to have certain characteristics without explicit measurement [3]. Specifically, previous work has investigated wireless network deployment in terms of gateway placement, channel assignment, and routing [4], [5]. However, each of these works focus on the deployment in WiFi bands without considering the white space bands. Moreover, the assumption of idle channels held in these models fails to match the infield spectrum utility, which could degrade the performance of a wireless network. These two issues are critical for designing an optimal network deployment and providing commercial wireless services to clients in any location.

Thus, the new opportunities created by white spaces motivate the following questions for wireless Internet carriers, which have yet to be addressed: (i) To what degree can white space bands reduce the network deployment cost of sparsely populated rural areas as opposed to comparable WiFi-only solutions? and (ii) Where along the continuum of user population densities do the white space bands no longer offer cost savings for wireless network deployments? In this paper, we perform a measurement study which considers the propagation characteristics and observed in-field spectrum availability of white space and WiFi channels to find the total number of access points required to serve a given user demand. Across varying population densities in representative rural and metropolitan areas, we compare the cost savings (defined in terms of number of access points reduced) when white space bands are not used. To do so, we first define the metric to quantify the spectrum utility in a given measurement location. With the in-field measured spectrum utility data in metropolitan and surrounding areas of Dallas-Fort Worth (DFW), we calculate the activity level in $\mathrm{WiFi}$ and white space bands. Second, we propose a measurement-driven framework to find the number of access points required for areas with differing population densities according to our measurement locations and census data. We then evaluate our measurement-driven framework, showing the band selection across downtown, residential and university settings in urban and rural areas and analyze the impact of white space and WiFi channel combinations on a wireless deployment in these representative scenarios.

The main contributions of our work are as follows:

- We perform in-field measurements of spectrum utilization in various representative scenarios across the DFW metroplex, ranging from sparse rural to dense urban areas and consider the environmental setting (e.g., downtown, residential, or university campus).

- We develop a measurement-driven Multi-band Access 
Point Estimation (MAPE) framework to jointly leverage propagation and spectrum availability of white space and WiFi bands for wireless access networks across settings.

- We analyze our framework under capacity and coverage constraints to show that, with white space bands, the number of access points can be greatly reduced from WiFi-only deployments by up to $1650 \%$ in rural areas.

- We quantify the impact of white space and WiFi channel combinations to understand the tradeoffs involved in choosing the optimal channel setting, given a certain number of available channels from multiple bands.

\section{Challenges And Problem Formulation}

In this section, we illustrate the challenges of band selection in wireless network deployments and formulate the problem of band selection in wireless network deployments jointly using $\mathrm{WiFi}$ and white space bands. Further, we present a measurement-driven framework for estimating the access point number to serve the traffic demand of a given population.

\section{A. White Space Opportunity and Challenge}

Wireless propagation refers to the signal loss characteristics when wireless signals are transmitted through the wireless medium. The strength of the received signal depends on both the line-of-sight path (or lack thereof) and multiple other paths that result from reflection, diffraction, and scattering from obstacles [6]. The widely-used Friis equation characterizes the received signal power $P_{r}$ in terms of transmit power $P_{t}$, transmitter gain $G_{t}$, receiver gain $G_{r}$, wavelength $\lambda$ of the carrier frequency, distance $R$ from transmitter to receiver, and path loss exponent $n$ according to [7]:

$$
P_{r}=P_{t}+G_{t}+G_{r}+10 n \log _{10}\left(\frac{\lambda}{4 \pi R}\right)
$$

Here, $n$ varies according to the aforementioned environmental factors with a value ranging from two to five in typical outdoor settings [8].

Despite sufficient levels of received signal, interference can cause channels to be unusable (e.g., due to high levels of packet loss) or unavailable (e.g., due to primary users in cognitive radios [9]). Prior work has worked to reduce interference levels via gateway deployment, channel assignment, and routing [4], [10]. The interference of a wireless network could be divided into two categories according to the interfering source: $(i)$ intra-network interference, caused by nodes in the same network, and (ii) inter-network interference, caused by nodes or devices outside of the network. Most of the existing works try to reduce the intra-network interference without regard to the inter-network interference [3]. However, the existence of inter-network interference becomes an important problem when considering the availability of white space bands. While theoretical models which describe inter-network interference exist, accurately characterizing a particular region must be done empirically.

When wireless devices operate in WiFi bands, the channel separation is relatively small (e.g., $5 \mathrm{MHz}$ for the $2.4 \mathrm{GHz}$ band). As a result, many works assume that the propagation characteristics across channels are similar. However, with the large frequency differences between $\mathrm{WiFi}$ and white space bands (e.g., multiple $\mathrm{GHz}$ ), propagation becomes a key factor in the deployment of wireless networks with both bands. Here, a frequency band is defined as a group of channels which have little frequency separation, meaning they have similar propagation characteristics. In this work, we consider the diverse propagation and activity characteristics for four total frequency bands: $450 \mathrm{MHz}, 800 \mathrm{MHz}, 2.4 \mathrm{GHz}$, and $5.2 \mathrm{GHz}$. We refer to the two former frequency bands as white space bands and the two latter frequency bands as WiFi bands. The differences in propagation and spectrum utilization create opportunities for the joint use of white space and WiFi bands in wireless access networks according to the environmental characteristics (e.g., urban or rural and downtown or residential) of the deployment location.

Typically, the deployment of wireless access networks is subject to coverage and capacity constraints for a given region. Coverage is defined with respect to the ability of clients to connect to access points within their service area. We use a coverage constraint ratio of $95 \%$ in this work for a target area [11]. Capacity is defined with respect to the ability of a network to serve the traffic demand of clients. Spatial reuse allows improved capacity but increases the cost of deploying a network by increasing the total number of access points required. Hence, for densely-populated areas, the greatest level of spatial reuse possible is often desired. In contrast, sparselypopulated rural areas have lower traffic demand per unit area. Thus, aggregating this demand with the use of lower frequencies via white space bands could be highly effective in reducing the total number of access points required to achieve similar coverage and capacity constraints. Moreover, since less TV channels tend to be occupied in sparsely-populated areas [12], a larger number of white space bands can be leveraged in these areas.

\section{B. Model and Problem Formulation}

As opposed to previous works such as [3], [10], [13], this paper focuses on reducing the inter-network interference for various population densities for wireless access networks which jointly employ WiFi and white space bands. We propose a measurement-driven framework to estimate the number of access points required for serving the traffic demand of a certain area. We assume an access point has a limited number of radios which operate on any channel of a fixed number of channels with the same antenna gain. Each radio on an access point operates with a classic protocol model [14]. We further assume that there is a given take rate and traffic demand for a given population (as specified in Section III).

For spectrum utility and resulting channel availability, we use a long-term measurement for each band. We define the percentage of sensing samples $\left(S_{\theta}\right)$ above an interference threshold $(\theta)$ over the total samples $(S)$ in a time unit as the activity level $(A)$ of inter-network interference:

$$
A=\frac{S_{\theta}}{S_{a}}
$$

The capacity of a clean channel is denoted by $C$. With the protocol model, the capacity of a channel with inter-network interference $C_{r}$ could be represented as the remaining free 


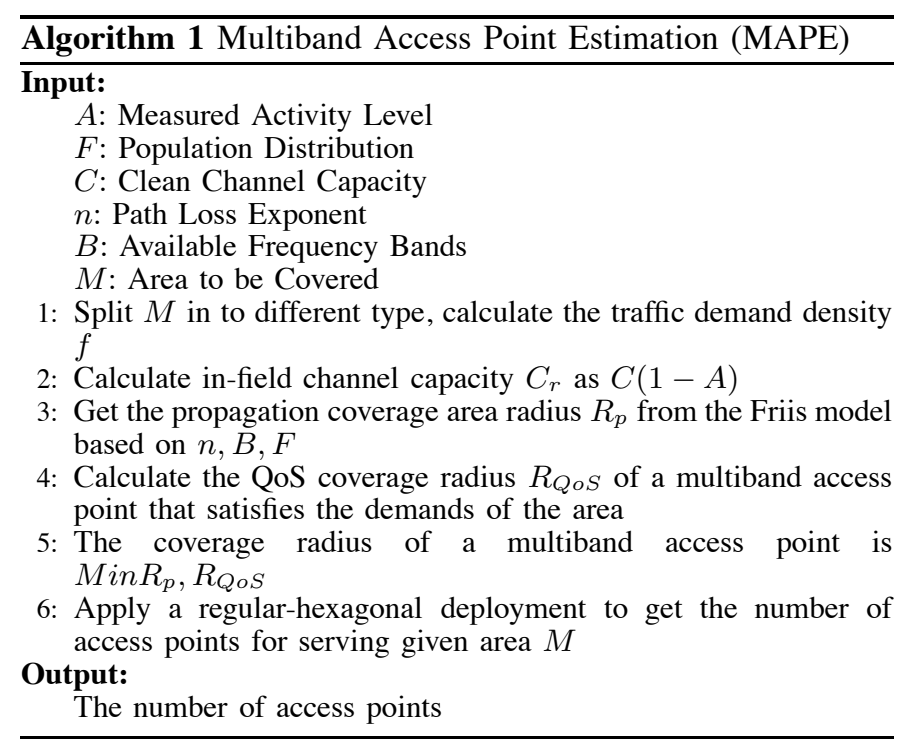

time of the channel capacity according to:

$$
C_{r}=C *(1-\bar{A})
$$

A network deployment should ideally provide network capacity equal to the demand of the service area to maintain the capacity constraint. The demand of a service area could be calculated as the summation of individual demands all over the service area $D_{a}=\sum_{p \in P} D_{p}$. Since household demand for the Internet has been previously characterized [15], $D_{a}$ could represent the population distribution $f$ and service area $k$ as $D_{a}=\sum_{f \in F, k \in K} \bar{D}_{p} * f * k$. The capacity constraint could be represented with an access point set $M$ according to:

$$
\sum_{m \in M} C_{r}^{m} \geq \sum_{f \in F, k \in K} \bar{D}_{p} * f * k
$$

At the same time, the wireless network must additionally satisfy the coverage constraint in the service area where the access points provide connectivity for client devices. Generally, a coverage of $95 \%$ is acceptable for wireless access networks [11].

In a joint white space and $\mathrm{WiFi}$ scenario, the activity level varies according to various interfering sources and the propagation characteristics induced by the environmental characteristics of the service area. A simple method with the least number of access points to cover an area is to use multiple orthogonal lower-frequency channels. However, the FCC limits white space band availability for data networks in most metropolitan areas in the United States [16]. Moreover, the number of channels in each band is limited. Too many lowerfrequency channels will cause high levels of intra-network interference for the network, which is out of our scope in this work. We assume that the cost of the network is proportional to the number of access points required for a given user demand (i.e., due to the cost of hardware and installation). Therefore, given a geographical region for a new network deployment, we build a measurement-driven framework called Multiband Access Point Estimation (MAPE) to compute the required number of access points.
In the space domain, the advantage of higher-frequency channels is the spatial reuse, while the lower-frequency channels provide greater levels of coverage. Generally, higher frequencies are more appropriate for populated areas, and lower frequencies are more appropriate for sparse areas. The temporal variation of spectrum utilization differs across bands. For an Internet service provider, the service quality which maps to the capacity constraint must be satisfied. Given a metropolitan area, the population distribution can be found according to census data [17]. Then, we can estimate the capacity demand of each type of area with the assumption that users will exhibit average demand. According to the population distribution, we split the area into different types, which compose the spatial input. Then, we use the measured activity level as the temporal input. We have an average channel capacity of each band according to the activity level. With the received signal strength threshold, the Quality-ofService-constrained coverage area of different types per channel, and the spatial reuse distance can be directly computed. Then, the maximum area an access point could cover can be calculated as the minimal area of the QoS-based coverage area and propagation coverage. Then, the transmission power is adjusted to fulfill the coverage restriction subject to the FCC regulations for maximum-allowable transmit power. A classic regular-hexagonal deployment process is employed to place the access points.

\section{EXPERIMENT AND ANALYSIS}

To evaluate the spectrum utility from in-field measurements, we perform experiments with an off-the-shelf wireless platform and mobile spectrum analyzer. According to the measured data, we apply our MAPE framework to analyze the role of white space and $\mathrm{WiFi}$ bands in the total access points required for a given deployment area and user demand.

\section{A. Experiment Design}

We employ a Linux-based 802.11 testbed, which includes a Gateworks 2358 board with Ubiquiti XR radios (XR9 at 900 $\mathrm{MHz}, \mathrm{XR} 2$ at $2.4 \mathrm{GHz}, \mathrm{XR} 5$ at $5.2 \mathrm{GHz}$ ) and a DoodleLabs DL475 radio at $450 \mathrm{MHz}$. We develop shell scripts which utilize tcpdump to enable the testbed to work as a sniffer, recording all 802.11 packets. However, since the Gateworks platform only updates its estimate of received signal strength upon the reception of a new packet (and not all relevant channel activity is 802.11 based), we employ a spectrum analyzer to form a notion of inter-network interference with finer granularity. Hence, we also use a Rohde \& Schwarz FSH8 portable spectrum works from $100 \mathrm{KHz}$ to $8 \mathrm{GHz}$. The portable spectrum analyzer is controlled by a Python script on a laptop to measure the received signal strength.

To the best of our knowledge, there is no readily available mobile, multiband antenna from $450 \mathrm{MHz}$ to $5.2 \mathrm{GHz}$ on the market. Thus, we use a 700-MHz mobile antenna to perform in-field measurements. We then normalize the mobile antenna performance across bands with indoor experimentation. To do so, we use a Universal Software Radio Peripheral (USRP) $\mathrm{N} 210$ to generate signals at $450 \mathrm{MHz}, 800 \mathrm{MHz}$, and $2.4 \mathrm{GHz}$. We feed the USRP signals directly to a spectrum analyzer and adjust the configuration of USRP to make the received signal 


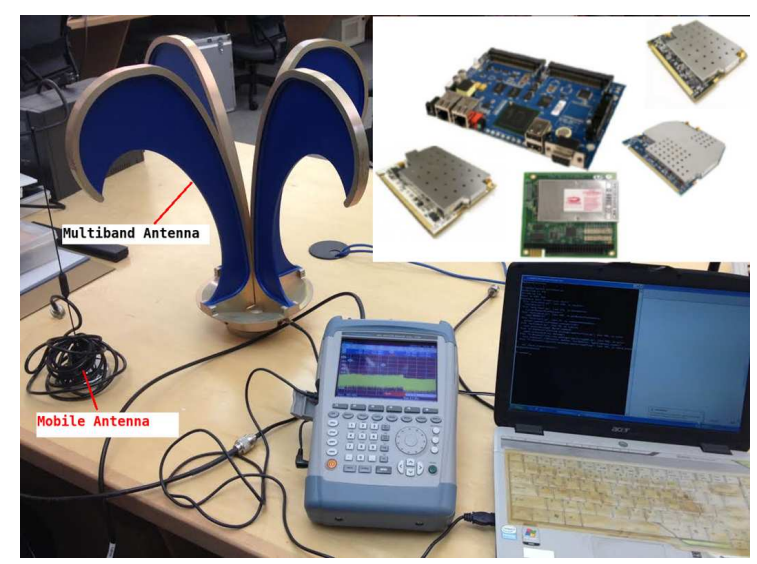

Fig. 1. Multiband Measurement Platform

strength the same as the $5.2 \mathrm{GHz}$ signal from Gateworks 2358 with a XR5 radio. Then, we connect the signal source to a fixed multiband antenna (QT 400 Quad Ridge Horn Antenna) and measure the received signal at a fixed distance with the $700 \mathrm{MHz}$ antenna and antennas for different bands to obtain the antenna loss for each band. We adjust the received signal strength collected via the $700-\mathrm{MHz}$ mobile antenna according to the normalization.

Our experimental platform is shown in Figure 1. The mobile spectrum analyzer records 32 samples per second on each band under test with appropriate time stamps. The Gateworks sniffer platform also records all the received WiFi packets according to their time stamps. The duplicate samples in WiFi bands from spectrum analyzer and Gateworks are deleted according overlapping time stamps. Accordingly, we calculate the activity level in WiFi bands. The activity level of white space bands is calculated solely based upon the spectrum analyzer measurements.

Figure 2 depicts a map of the available white space channels with markers where we performed measurements in North Texas. To be representative of a broad range of community types, we consider populations of approximately 25 times one another according to the 2010 U.S. Census, Millsap (500), Weatherford $(25 \mathrm{~K})$, and Dallas $(1.25 \mathrm{M})$. We have collected measurements at multiple types of locations in Dallas, including a downtown area, a residential area, and a university campus. In Weatherford and Millsap, we monitor wireless activities in three locations for 45 continuous minutes on a weekday in downtown, residential, and non-residential areas. Then, we post-process the data to calculate the activity level of each band in each location. First, we parse the SNR from the data logs via Perl scripts. Second, we merge the data from the two platforms according to their respective time stamps and calculate the activity level of each band across these locations. The activity level is then included in our framework as input parameter.

\section{B. Results and Analysis}

In this subsection, we discuss our measurements results and leverage our MAPE framework to analyze the influence of white space channels across areas with different population densities. As an initial experiment, we perform a drive test from Dallas to Weatherford with cruise control set to $60 \mathrm{MPH}$

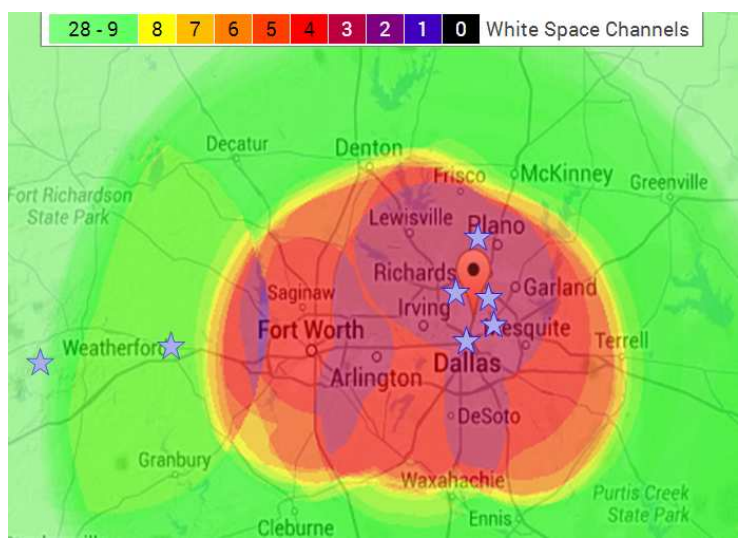

Fig. 2. White Space Channels in DFW Metropolitan and Surrounding Areas.
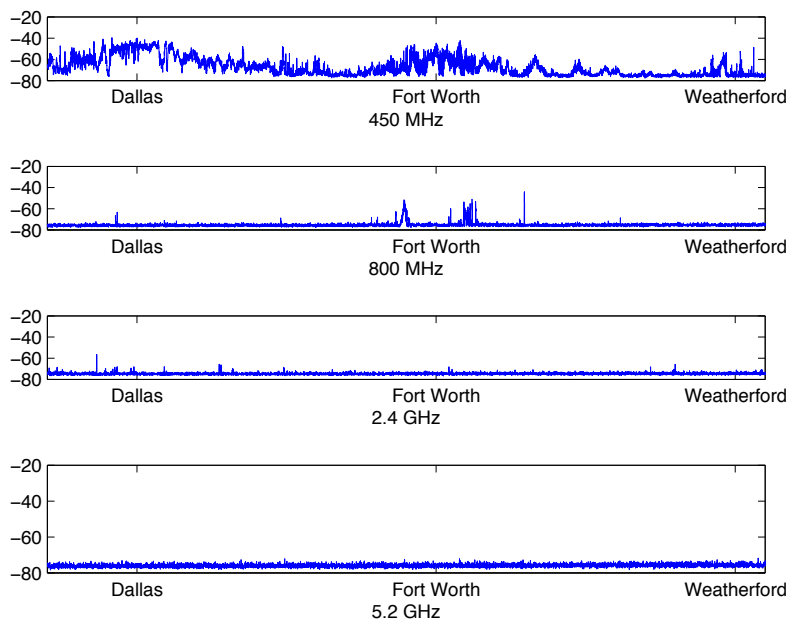

Fig. 3. Spectrum Activity in DFW Metropolitan and Surrounding Areas.

while on the highway. The result of the in-field spectrum drive test is shown in Figure 3 according to the location and time of the measurement. The measured activity via RSSI of 450 $\mathrm{MHz}$ is high in downtown Dallas and Fort Worth but has less signal activity in the urban and rural area between these city centers. The low activity detected in the WiFi bands is due to the distance from the highway being typically larger than the propagation range of predominantly indoor wireless routers. Our initial in-field measurement matches the FCC restrictions (shown in Figure 2) with less channels available translating to greater spectrum utilization by TV stations. The drive test also shows that the spectrum utilization is roughly proportional to the population density in Figure 3. We use the measurements collected at more fixed locations as marked on the map for the activity level calculation.

The activity level calculated with our measurements are shown in Table I. Dallas, the city with the greatest population in North Texas, has the highest activity level in most of the measured bands, especially at $450 \mathrm{MHz}$. The Dallas urban measurements are taken from the SMU campus, two neighborhoods, and a densely-populated suburb (Plano). Our measurements indicate that $2.4 \mathrm{GHz}$ has a higher activity level in urban area than the measured downtown area. Most schools and their neighborhoods are covered by $\mathrm{WiFi}$, which contributes to the high activity level at $2.4 \mathrm{GHz}$ and $5.2 \mathrm{GHz}$. In Weatherford, all the bands have lower activity levels than 


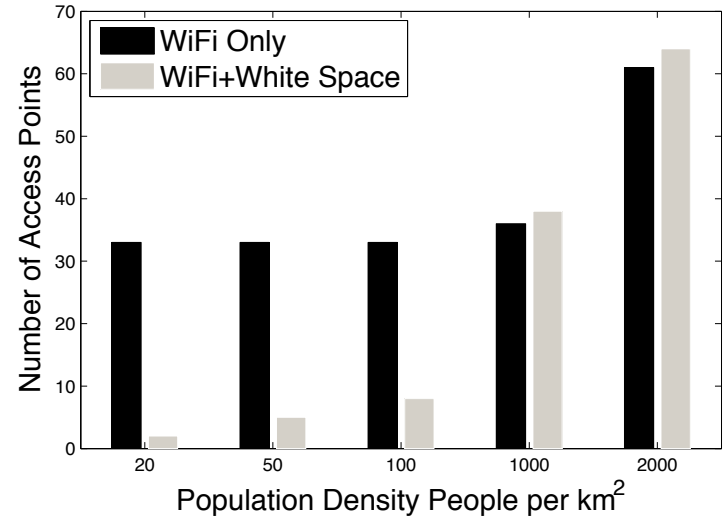

Fig. 4. Number of Access Points Needed for a $13 \mathrm{~km}$ x13 km Area. in Dallas. A peculiarity in the measurements can be seen by the sparse area in Weatherford having more activity than the other regions for $450 \mathrm{MHz}$. This can be explained due to the measurement location being on the East of Weatherford (closer to Fort Worth, which has a population of approximately $750 \mathrm{k}$ ). Millsap is a typical sparse rural area with approximately 500 total residents. The activity levels across all the bands are lower than in Dallas and Weatherford. In the $450 \mathrm{MHz}$ band, the activity level decreases much faster than in other bands in Dallas and Weatherford.

We use the measurement-based activity levels shown in Table I in our framework presented in Algorithm 1. We specifically use the Millsap sparse area, Millsap downtown, Weatherford residential, Dallas residential, and Dallas downtown measurements as inputs of activity level for a given population density. We then calculate the number of access points for covering a $13 \mathrm{~km} \times 13 \mathrm{~km}$ area, varying the population density. The output is shown in Figure 4.

In the calculation, we set the demand requested per user as $2 \mathrm{Mbps}$ with the population density of $20,50,100,1000$, and 2000 users per square kilometer. We assume $30 \%$ of the residents will use this service (i.e., the take rate is 30 $\%$ ), the maximum transmit power is $30 \mathrm{dBm}$, and a path loss exponent of 3.5 [18]. From Equation 1, we see that the propagation range is proportional to the wavelength with 450 $\mathrm{MHz}$ having a propagation range of 11.6 times that of 5.2 GHz. We adopt an 802.11n maximum data rate of $600 \mathrm{Mbps}$. For the WiFi+White Space scenario, we use 3 channels in each of the $450 \mathrm{MHz}, 2.4 \mathrm{GHz}$ and $5.2 \mathrm{GHz}$ bands. For the WiFi Only scenario, we assume 6 channel in the 2.4-GHz band, and 3 channels in the $5.2-\mathrm{GHz}$ band since $2.4 \mathrm{GHz}$ has larger propagation range than $5.2 \mathrm{GHz}$. Each of these scenarios have the same channels in total (9). As shown in Figure 4, with the same number of channels, WiFi+White Space reduces the number of access points by $1650 \%$ compared to the WiFi Only scenario in the 20 people per square $\mathrm{km}$ scenario, $660 \%$ in the 50 people per square $\mathrm{km}$, and $412.5 \%$ in the 100 people per square $\mathrm{km}$ scenario. The large propagation range of the white space bands is approximately 10 times that of the WiFi bands, creating an opportunity for greater coverage. However, as the population density increases, due to the capacity constraint of servicing users in the area, the lower-frequency white space bands lose their advantage of larger communication range due to the reduction in achievable spatial reuse. At the same time, the activities of other signal sources, such as TV stations in downtown areas, reduce the capacity of white space bands. As a result, the WiFi+White Space scenario performs worse than the WiFi Only scenario. If we were to count the intra-network interference (out of scope), the situation could become even worse. Moreover, FCC has stricter policies on white spaces in urban areas. Fewer channels are available in these areas, which makes WiFi a better option for dense areas.

To understand the influence of band combinations on network deployments, we calculate the number of access points in the area when selecting 500 people per square $\mathrm{km}$ with a downtown Weatherford spectrum utilization and 1500 people per square $\mathrm{km}$ with a residential Dallas spectrum utilization. We assume the total number of channels is 12 . We use the same setup as the previous experiment.

\begin{tabular}{|c|c|c|c|}
\hline \multirow{3}{*}{ No. of Bands } & Bands Combination & \multicolumn{2}{|c|}{ No. of AP } \\
\cline { 2 - 4 } & $(\mathrm{Hz})$ & $\begin{array}{c}500 \\
p p l / \mathrm{km}^{2}\end{array}$ & $\begin{array}{c}1500 \\
p p l / \mathrm{km}^{2}\end{array}$ \\
\hline \multirow{4}{*}{1} & $450 \mathrm{M}$ & 12 & 35 \\
\cline { 2 - 4 } & $800 \mathrm{M}$ & 10 & 30 \\
\cline { 2 - 4 } & $2.4 \mathrm{GHz}$ & 33 & 37 \\
\cline { 2 - 4 } & $5.2 \mathrm{G}$ & 193 & 193 \\
\hline \multirow{4}{*}{2} & $450 \mathrm{M}, 800 \mathrm{M}$ & 11 & 32 \\
\cline { 2 - 4 } & $450 \mathrm{M}, 2.4 \mathrm{G}$ & 23 & 36 \\
\cline { 2 - 4 } & $450 \mathrm{M}, 5.2 \mathrm{G}$ & 23 & 69 \\
\cline { 2 - 4 } & $800 \mathrm{M}, 2.4 \mathrm{G}$ & 20 & 33 \\
\cline { 2 - 4 } & $800 \mathrm{M}, 5.2 \mathrm{G}$ & 20 & 59 \\
\cline { 2 - 4 } & $2.4 \mathrm{G}, 5.2 \mathrm{G}$ & 33 & 73 \\
\hline \multirow{3}{*}{3} & $450 \mathrm{M}, 800 \mathrm{M}, 2.4 \mathrm{G}$ & 16 & 33 \\
\cline { 2 - 4 } & $450 \mathrm{M}, 800 \mathrm{M}, 5.2 \mathrm{G}$ & 16 & 48 \\
\cline { 2 - 4 } & $450 \mathrm{M}, 2.4 \mathrm{G}, 5.2 \mathrm{G}$ & 33 & 53 \\
\cline { 2 - 4 } & $800 \mathrm{M}, 2.4 \mathrm{G}, 5.2 \mathrm{G}$ & 30 & 49 \\
\hline 4 & $450 \mathrm{M}, 800 \mathrm{M}, 2.4 \mathrm{G}, 5.2 \mathrm{G}$ & 21 & 44 \\
\hline
\end{tabular}

TABLE II

Channel COMbinations FOR 500 AND 1500 Population DENSITY SCENARIOS

In Table II, we compare the number of access points with 12 channels through all the possible combinations of bands. Since purchasing and deploying access points is the primary cost of a wireless infrastructure, to simplify the calculation, we only count the number of access points as the network's cost. When all the channels are in the same band, as the frequency goes up, more access points are needed to serve the area due to the limited propagation range. However, 450 $\mathrm{MHz}$ does not outperform $800 \mathrm{MHz}$ with a single band at both the 500 and 1500 people per square $\mathrm{km}$ cases because $450 \mathrm{MHz}$ channels have larger measured activity levels. White space band channels outperform WiFi bands by up to $1830 \%$ in the single band case with 500 people per square $\mathrm{km}$, but with 1,500 people per square $\mathrm{km}$, the cost reduction decreases to only $543 \%$. We now distribute an equal number of channels to two-band combinations and run the experiments with the same population densities and spectrum utilization. The results shows the white space band combination (450 and $800 \mathrm{MHz})$ performs better than WiFi only $(2.4$ and $5.2 \mathrm{GHz})$ by $200 \%$ and $128 \%$ with the people per square $\mathrm{km}$ of 500 and 1,500 , respectively. In fact, the white space only scenario (450 and $800 \mathrm{MHz}$ ) has almost the same performance as the scenarios with one white band and one WiFi band $(450 \mathrm{MHz}$ and 2.4 $\mathrm{GHz} ; 800 \mathrm{MHz}$ and $2.4 \mathrm{GHz}$ ) with 1,500 people per square $\mathrm{km}$. However, with 500 people per square $\mathrm{km}$, the white space only scenario is much better than any other two-band 


\begin{tabular}{|c|c|c|c|c|c|c|c|c|c|}
\hline Bands & \multicolumn{3}{|c|}{ Dallas } & \multicolumn{3}{|c|}{ Weatherford } & \multicolumn{3}{|c|}{ Millsap } \\
\hline Area Type & Downtown & Residential & Suburban & Downtown & Residential & Sparse & Downtown & Residential & Sparse \\
\hline $450 \mathrm{MHz}$ & 24.37 & 25.83 & 23.77 & 6.05 & 12.50 & 14.03 & 7.00 & 0.07 & 0.02 \\
\hline $800 \mathrm{MHz}$ & 4.40 & 16.49 & 4.77 & 5.22 & 5.07 & 4.43 & 3.87 & 4.20 & 3.60 \\
\hline $2.4 \mathrm{GHz}$ & 15.87 & 34.95 & 2.60 & 2.03 & 2.03 & 2.77 & 2.07 & 1.60 & 0.80 \\
\hline $5.2 \mathrm{GHz}$ & 19.70 & 35.46 & 1.53 & 1.93 & 1.93 & 1.33 & 1.27 & 2.07 & 2.10 \\
\hline
\end{tabular}

TABLE I

ACTIVITy LeVEl in Multiple Locations

combination. White space channels provide up to $87.5 \%$ cost reduction in three-band combination scenarios with 500 people per square $\mathrm{km}$, and up to $33.3 \%$ with 1,500 people per square $\mathrm{km}$. With four bands, the number of access points required does not reduce using white space bands.

From Figure 4 and Table II, we show that as the population density increases, the reduction in number of access points required to meet the same demand diminishes. Note that a more optimal allocation of channels in different bands could offer further cost reductions. We further show that as population and spectrum utilization increase, at some point, the performance of white space only scenario could be the same as a combination of white space and WiFi bands.

\section{RELATED WORK}

Prior work in wireless network deployment has focused extensively on solving gateway placement, channel assignment, and routing problems to reduce the interference generated inside the network [4], [19], [20]. Unfortunately, few works in network deployment notice the interference across networks. Some cognitive radio works discuss this inter-network interference, but most of them focus on point-to-point communication other than taking a network-wide view [21].

With new FCC regulations on the use of white space bands, there are two factors to consider with such bands: large propagation range and existing inter-network interference from TV stations and other devices such as microphones [22] [24]. Prior work does not specifically study the benefits of jointly using white space and WiFi bands in the deployment of wireless access networks [25]. Additionally, prior work related to white spaces target opportunistic media access. However, the application of white spaces across diverse population densities has not been fully explored.

Finally, some works discuss the propagation variation in both WiFi bands and white space bands. For example, Robinson et al. models the propagation variation at the same band in terrain domain [11]. Another work proposes a databasedriven framework for designing a white space network with database of primary user (TV station) locations and channel occupation [26]. However, these works do not jointly study the influence of white space and WiFi bands on network deployment according to their resulting propagation variation and spectrum utilization.

\section{CONCLUSION}

In this paper, we jointly considered the use of $\mathrm{WiFi}$ and white space bands for deploying wireless access networks across a broad range of population densities. To consider network deployment costs, we proposed a Multiband Access Point Estimation framework to find the number of access points required in a given region. We then performed spectrum utilization measurements in the DFW metropolitan and surrounding areas to drive our framework and find the influence of white spaces on network costs in these representative areas. Through extensive analysis across varying population density and channel combinations across bands, we show that white space bands can reduce the number of access points by $1650 \%$ and $660 \%$ in rural and sparse urban areas, respectively. However, the same cost savings are not achieved in dense urban and downtown type area. Finally, we investigate different band combinations in two population densities to show that greater access to white space channels have greater total savings of mesh nodes when the total number of channels used in the network is fixed (i.e., given a total number of allowable WiFi and white space channels). As the population and spectrum utilization increase, the cost savings of white space bands diminish to the point that WiFi-only channel combinations can be optimal. In the future, we will consider the heterogeneous access points and traffic demand scenarios in wireless network deployments.

\section{ACKNOWLEDGMENTS}

We would like to thank the anonymous reviewers for their valuable feedback, which helped in improving the presentation of the paper. This work was in part supported by NSF grants: CNS-0958436, CNS-1150215, and CNS-1320442.

\section{REFERENCES}

[1] C. Inc., "Fcc certifies carlson wireless technologies tv white space radio," http://www.carlsonwireless.com/rural-connect-press-release.html, 2014.

[2] C. A. Balanis, Antenna theory: analysis and design. John Wiley \& Sons, 2012.

[3] W. Si, S. Selvakennedy, and A. Y. Zomaya, "An overview of channel assignment methods for multi-radio multi-channel wireless mesh networks," Journal of Parallel and Distributed Computing, vol. 70, no. 5, pp. 505-524, 2010.

[4] B. He, B. Xie, and D. P. Agrawal, "Optimizing deployment of internet gateway in wireless mesh networks," Computer Communications, vol. 31, no. 7, pp. 1259-1275, 2008.

[5] M. K. Marina, S. R. Das, and A. P. Subramanian, "A topology control approach for utilizing multiple channels in multi-radio wireless mesh networks," Computer Networks, vol. 54, no. 2, pp. 241-256, 2010.

[6] J. B. Andersen, T. S. Rappaport, and S. Yoshida, "Propagation measurements and models for wireless communications channels," IEEE Communications Magazine, vol. 33, no. 1, pp. 42-49, 1995.

[7] H. T. Friis, "A note on a simple transmission formula," vol. 34, no. 5, pp. 254-256, May 1946.

[8] T. Rappaport, Wireless Communications, Principles \& Practice. Prentice Hall, 1996.

[9] S. Haykin, "Cognitive radio: brain-empowered wireless communications," Selected Areas in Communications, IEEE Journal on, vol. 23, no. 2, pp. 201-220, 2005.

[10] J. Tang, G. Xue, and W. Zhang, "Interference-aware topology control and QoS routing in multi-channel wireless mesh networks," in $A C M$ MobiHoc, 2005.

[11] J. Robinson, M. Singh, R. Swaminathan, and E. Knightly, "Deploying mesh nodes under non-uniform propagation," in IEEE INFOCOM, 2010.

[12] "Microsoft research white space database," http://whitespaces.cloudapp.net/Default.aspx, 2013. 
[13] J. Yuan, Z. Li, W. Yu, and B. Li, "A cross-layer optimization framework for multihop multicast in wireless mesh networks," IEEE JSAC, vol. 24 no. 11 , pp. 2092-2103, 2006.

[14] P. Gupta and P. R. Kumar, "The capacity of wireless networks," IEEE Trans. on Information Theory, vol. 46, no. 2, pp. 388-404, 2000.

[15] G. Rosston, S. Savage, and D. Waldman, "Household demand for broadband internet service," Communications of the ACM, vol. 54, no. 2 , pp. 29-31, 2011.

[16] "Google spectrum database," http://goo.gl/NnIFXQ, 2013.

[17] "People and households," http://www.census.gov/people/, 2014.

[18] R. Meikle and J. Camp, "A global measurement study of contextbased propagation and user mobility," in Proceedings of the 4th ACM international workshop on Hot topics in planet-scale measurement. ACM, 2012, pp. 21-26.

[19] K. N. Ramachandran, E. M. Belding-Royer, K. C. Almeroth, and M. M. Buddhikot, "Interference-aware channel assignment in multi-radio wireless mesh networks." in IEEE INFOCOM, 2006.

[20] I. F. Akyildiz, W.-Y. Lee, M. C. Vuran, and S. Mohanty, "Next generation/dynamic spectrum access/cognitive radio wireless networks: a survey," Computer Networks, vol. 50, no. 13, pp. 2127-2159, 2006.

[21] D. Cabric, S. Mishra, and R. Brodersen, "Implementation issues in spectrum sensing for cognitive radios," in Signals, Systems and Computers, 2004. Conference Record of the Thirty-Eighth Asilomar Conference on, vol. 1. Ieee, 2004, pp. 772-776.

[22] "Fcc white space," http://www.fcc.gov/topic/white-space, 2012.

[23] P. Cui, H. Liu, J. He, O. Altintas, R. Vuyyuru, D. Rajan, and J. Camp, "Leveraging diverse propagation and context for multi-modal vehicular applications," in IEEE WiVeC, 2013.

[24] P. Bahl, R. Chandra, T. Moscibroda, R. Murty, and M. Welsh, "White space networking with WiFi like connectivity," ACM SIGCOMM, vol. 39 , no. 4 , pp. 27-38, 2009.

[25] I. F. Akyildiz, X. Wang, and W. Wang, "Wireless mesh networks: a survey," Computer networks, vol. 47, no. 4, pp. 445-487, 2005.

[26] R. Murty, R. Chandra, T. Moscibroda, and P. Bahl, "Senseless: A database-driven white spaces network," Mobile Computing, IEEE Transactions on, vol. 11, no. 2, pp. 189-203, 2012. 